\title{
Tourisme, travail, migration : interrelations et logiques mobilitaires
}

\section{Olivier Dehoorne}

\section{(2) OpenEdition \\ 1 Journals}

Édition électronique

URL : https://journals.openedition.org/remi/1676

DOI : $10.4000 /$ remi.1676

ISSN : $1777-5418$

Éditeur

Université de Poitiers

\section{Édition imprimée}

Date de publication : 1 mars 2002

Pagination : 7-36

ISBN : 2-911627-30-X

ISSN : 0765-0752

\section{Référence électronique}

Olivier Dehoorne, "Tourisme, travail, migration : interrelations et logiques mobilitaires », Revue européenne des migrations internationales [En ligne], vol. 18 - $n^{\circ} 1$ | 2002, mis en ligne le 09 juin 2006, consulté le 14 avril 2022. URL : http://journals.openedition.org/remi/1676 ; DOI : https://doi.org/ 10.4000/remi. 1676

Ce document a été généré automatiquement le 14 avril 2022.

(c) Université de Poitiers 


\title{
Tourisme, travail, migration : interrelations et logiques mobilitaires
}

\author{
Olivier Dehoorne
}

1 Le lieu touristique n'existe qu'à travers les mobilités et le marché touristique est au cœur de trajectoires migratoires variées et renouvelées dans un contexte international contraignant.

2 Les mobilités croissantes de nos sociétés reflètent les transformations de modes de vie. À travers les déplacements de loisirs à l'échelle internationale se dessinent de nouveaux rapports entre les modes et les lieux de production et de consommation. Si ces lieux localisés sur les marges des régions les plus riches (les côtes nord de la Méditerranée et des Caraïbes) continuent à dominer le marché touristique, la croissance soutenue de l'activité à l'échelle mondiale s'accompagne d'une plus grande dispersion des flux et donc des recettes, expliquant localement l'importance des espoirs suscitée dans des pays en difficulté.

Deux types de situations sont donc à distinguer: celle des régions de tourisme de masse, où l'activité plus ancienne se caractérise par une internationalisation du marché depuis les années soixante et celle des régions émergentes où se construisent les nouveaux lieux touristiques. Le phénomène migratoire est plus frappant dans ce second cas mais il n'en demeure pas moins une réalité sur tous les marchés; dans les lieux où l'activité est mieux structurée, des déplacements saisonniers sont devenus des migrations définitives - faisant du tourisme une activité peuplante - tandis que d'autres migrations temporaires alimentent le marché.

4 Le tourisme est désormais une réalité économique établie qui se mesure par ses recettes, ses emplois et par la force des images et des représentations générées (Claval 1995). "Même très imparfaitement mesurés,... ils (les flux touristiques) constituent aujourd'hui les déplacements humains les plus massifs et l'un des aspects les plus visibles de la mondialisation des échanges. Le tourisme est probablement devenu la première activité du monde ${ }^{1}$. Dès lors, il n'est guère surprenant que pour bien des responsables politiques et 
économiques, le secteur touristique apparaisse désormais vital, ou pour le moins prometteur, et ceci à des échelles fort variées » Dewailly et Flament (1993).

5 Les interrelations entre flux touristiques et flux migratoires sont de plus en plus complexes et intenses. Les flux se stimulent réciproquement, et les touristes s'impliquent davantage dans ces lieux touristiques, lieux que les migrants utilisent également dans leur logique de déplacement vers les régions les plus développées.

6 La réflexion sur les enjeux migratoires du marché touristique doit être replacée dans le contexte actuel qui vise à contrôler les migrations vers le Nord en contrepartie d'un développement dans les pays d'origine. Les volontés politiques qui guident la création de lieux touristiques dans ces pays doivent être mises en perspective avec le paradigme de la croissance (Guengant, 1996) comme élément de réponse au contrôle des flux migratoires.

7 Pour conduire cette étude, nous proposons dans un premier temps quelques éclairages sur les concepts de migration, de tourisme et de mobilité. La réflexion sur les interrelations entre ces flux requiert ensuite une analyse des marchés touristiques. C'est en s'interrogeant sur leur fonctionnement et leurs logiques que pourront être identifiées les différentes formes de mobilités et les stratégies originales qui se développent à partir de ces lieux.

Migration, tourisme : deux formes dans un même système des mobilitésMigrations, tourisme, mobilités : évolution des concepts

8 Les mobilités ne se réduisent pas aux mouvements migratoires et le tourisme n'est pas une migration même si l'expression "migration touristique " est parfois employée (Dewailly et Flament, 1993) : le touriste quitte temporairement son lieu de vie habituel alors que le migrant change de lieu de vie, il y a rupture. On a pu parler de "grande migration d'été des Français» (Cribier, 1969) pour qualifier les départs des citadins partagés entre les retours saisonniers au « pays » et les premiers déplacements massifs des vacanciers. Le tourisme est une des composantes de la mobilité, mais non une migration au sens strict, il ne peut donc pas être intégré à l'ensemble des flux migratoires ${ }^{2}$.

9 Reprenant la définition proposée par la Division Démographique des Nations Unies, il convient de réserver l'emploi du terme de migration à une forme particulière de mobilité : "les déplacements exceptionnels, entraînant l'installation durable dans un lieu autre que le lieu d'origine, et s'accompagnant d'un changement de lieu de résidence habituelle». Thumerelle (1986, p. 13) insiste sur «le franchissement d'un certain nombre de seuils de rupture: caractère irréversible ou de longue durée du déplacement, distance entre points de départ et d'arrivée suffisante pour amener sinon une rupture totale du moins une modification profonde dans l'espace de vie habituel du migrant ».

10 Les migrations se distinguent des navettes (déplacements quotidiens ou hebdomadaires), réalisées dans un espace de vie habituel. Elles correspondent à des déplacements qui restent exceptionnels dans l'existence des individus, marquant un départ définitif, ou pour une période plus ou moins longue, de son espace de vie habituel, au profit d'un autre espace qui devient un nouvel espace de vie. Mais ces pratiques évoluent, la rupture avec le milieu d'origine n'est plus aussi radicale, les migrants s'organisent au sein d'un «territoire circulatoire» (Tarrius, 1992). Les spécialistes des migrations parlent de "circulation migratoire " pour qualifier cette situation d'allers et de retours permanents (Domenach et Picouet, 1987). Cette réalité pose de sérieuses difficultés sur le plan statistique, les définitions classiques étant 
inadaptées : chaque fois qu'un migrant circule « entre ici et là-bas », il est comptabilisé comme touriste.

Mobilités et motivations

11 Le tourisme international franchit des frontières comme les migrants internationaux mais l'intentionnalité n'est pas la même. Le tourisme concerne des mobilités faites pour le plaisir; à des fins de loisirs, de jeu, de découverte. Ces déplacements décidés, contrôlés, maitrisés sont l'occasion de vivre des moments privilégiés de recréation (Knafou et alii., 1997). Le changement de lieu de vie est temporaire. Ces voyages impliquent un investissement financier sans perspective professionnelle. Nul ne peut imposer un lieu de séjour, le futur touriste peut recevoir des informations erronées, être influencé, abusé en raison de son empressement ou de son ignorance, mais dans tous les cas de figure, la décision finale relève de sa seule volonté.

Les motivations sont capitales pour distinguer les différentes formes de mobilités. Le degré de liberté est variable selon les types de déplacements (avec le cas extrême du réfugié). Si la part de liberté demeure dans le cas des migrations elle peut-être considérablement amoindrie par des déterminants économiques, familiaux, sociaux, politiques. Pour le tourisme, elle est entière.

13 Le déplacement est obligatoire pour faire du tourisme; l'absence du domicile habituel est, par définition, supérieure à vingt-quatre heures. Ce déplacement doit se faire hors de son cadre de vie habituel, quotidien, ce qui n'est pas le cas pour les loisirs qui peuvent se réaliser avec ou sans déplacement (les loisirs à domicile). Le déplacement de proximité (loisirs de proximité) s'effectue à la journée, l'excursionniste peut dans ce cadre côtoyer des touristes. Le « tourisme virtuel », la découverte d'un ailleurs à travers la virtualité (Internet ou cédérom), relève du domaine des loisirs. Le déplacement et la durée sont donc essentiels pour distinguer tourisme et loisir.

14 La limite supérieure pour un séjour touristique est d'un an pour les organismes officiels de recensement de l'activité, en fait au-delà de trois mois, le visiteur ne peut plus être considéré comme touriste, il devient un "résident» même si son visa reste touristique ${ }^{3}$.

15 Le touriste quitte donc temporairement son lieu de vie habituel pour aller vivre ailleurs, dans un ou des lieux situés hors de sphère de la vie quotidienne, il effectue "un changement de place» et, par «extension géographique», un changement "d'habiter" (Duhamel et Sacareau, 1998). "Le déplacement opère une discontinuité qui permet un autre mode d'habiter voué à la seule recréation » (Knafou et al., $1997: 198$ ).

Vers le concept de mobilité

16 Dans son acceptation la plus large, le concept de mobilité spatiale englobe l'ensemble des «déplacements dans l'espace physique, d'individus ou de groupes d'individus, quelle que soit la durée et la distance de ces déplacements » (Courgeau, 1988).

17 Les logiques des migrants comme celles des touristes évoluent, se diversifient et se complexifient en s'organisant à l'échelle planétaire (Simon, 1998). Les schémas d'identification classiques sont brouillés : l'émigré est devenu un migrant, il ne s'agit plus de transférer une sédentarité, un autre enracinement mais bien de garder sa mobilité et sa capacité à être mobile (« la compétence de mobilité »; Lévy, 2000), et d'utiliser ses connaissances des différentes sociétés pour un tirer un avantage lui permettant de développer une stratégie personnelle. «Cette compétence peut passer par la possession de moyens matériels, notamment financiers, à travers l'accès aux moyens de 
déplacement et, plus généralement, grâce à la constitution d'un réseau de lieux fréquentés (habitat, emploi, etc.) qui sont eux-mêmes en bonne position dans l'espace des accessibilités » (Lévy, $2000: 159$ ).

De la même façon, les résidents issus de sociétés plutôt sédentaires, sans tradition migratoire, découvrent de nouveaux lieux de vie à l'occasion de séjours touristiques plus fractionnés et plus fréquents. La connaissance qu'ils tirent de ces expériences a un impact grandissant dans leur vie quotidienne. La distinction entre le quotidien et l'occasionnel, qui hier encore pouvait être exceptionnel (prendre un avion par exemple), n'a plus la même portée. Les individus prennent conscience des compétences qu'ils peuvent valoriser dans le pays d'accueil avec les éventuels avantages financiers et surtout les améliorations possibles en terme de qualité de vie. Ce transfert d'activité, partiel ou complet, peut conduire du tourisme à une résidence alternée. On parle alors de multi-résidentialité ou de "polyspatialité » (Viard, 1994). Nous entrons ici dans le champ des «mobilités post-migratoires " qui s'effectuent dans un système-monde où ces mobilités intenses proviennent de régions inter-reliées, connectées. Les transactions se font en anglais, les règlements en dollars et parfois en euros. Les systèmes de références sont connus de chacun, et chacun évolue avec sa logique territoriale. Face à la réalité de ces lieux de vie multiples, Knafou propose de «revisiter le concept d'espace de vie " (Knafou, 2000: 92) pour "considérer le continuum de nos vies, tant dans l'espace que dans le temps». Les itinéraires circulatoires des individus qui accumulent des expériences et de nouvelles compétences spatiales au sein d'un espace de vie élargie, non plus confiné à un espace routinier, du quotidien, s'articulent désormais autour de plusieurs lieux de vie.

Il convient de donner au concept de mobilité une réelle dimension qui ne se bornerait pas à faire l'inventaire des différentes formes de déplacement sans y intégrer la dimension sociale. "On peut définir la mobilité comme la relation sociale au changement de lieu, c'est à dire l'ensemble des modalités par lesquelles les membres d'une société traitent la possibilité qu'eux-mêmes ou d'autres occupent successivement plusieurs localisations " (Lévy, 2000 , p. 155). La mobilité a plus de portées qu'un simple déplacement (comme les navettes quotidiennes), il faut s'interroger sur la signification sociale de ce mouvement. Mobilités et inégalités dans le système-monde

Dans le contexte de l'économie mondiale libérale, la libre circulation n'a pas le même sens pour tous: si les capitaux, les marchandises, les informations et les touristes circulent toujours plus vite et plus facilement, les travailleurs des pays les moins développés qui n'ont que leur force et leur jeunesse à vendre sont confrontés à un contrôle croissant. Après les migrations aisées des décennies de fortes croissances des puissances occidentales, les crises économiques successives ont engagé un ralentissement des flux migratoires. Au début des années quatre-vingt, Thumerelle retenait l'estimation de "une vingtaine de millions le nombre des travailleurs étrangers dans les pays d'accueil » pour des flux annuels de l'ordre d'un million d'actifs échangés depuis les pays d'émigration nette vers les pays d'immigration nette. Et le double pour " obtenir les effectifs réellement transférés puisque les membres de leurs familles accompagnent ou rejoignent les travailleurs" (Thumerelle, 1986, p. 245). "La tendance lourde des migrations stricto sensu - avec changement de résidence - semble aller en décroissant, puisque le taux d'émigration (...) a été divisé par deux depuis le début de ce siècle, passant de $5 \%$ environ avant la Première Guerre mondiale à $2 \%$ environ en cette fin $d u$ XXe siècle (119 millions en 1995 selon les calculs officiels des Nations Unies; en réalité, si l'on tient compte des 
clandestins 130 à 150 millions de migrants sur 6 milliards d'habitants)»(Simon, 1998, p. 61). Le contexte migratoire n'est plus le même. "Les migrations internationales actuelles peuvent apparaître, sur un certain plan, comme une forme résiduelle de la mise en place de l'espace habité, de l'œekoumène, mais leur signification actuelle constituerait plutôt une forme de réponse aux questions du monde actuel, à ses contradictions, à ses tensions comme à ses espérances... » (Simon, 1998, p. 61). Désormais les critères de sélection sont plus stricts, ce sont les plus instruits et les mieux formés qui circulent le plus aisément (Verquin, 1998).

21 À l'inverse, dans les pays les plus développés, les déplacements sont toujours plus nombreux. On y constate «la mobilité accrue de populations encore fondamentalement sédentaires» (Knafou, 1998). Et pendant qu'environ « un million d'actifs se déplace chaque année d'un pays à l'autre", les déplacements touristiques ne cessent de prendre de l'ampleur, sur le plan international comme dans les cadres nationaux: le nombre de touristes internationaux est passé de 25 millions en 1950 à 457 millions en 1990 pour atteindre 550 millions en $1995^{4}$. Soit une croissance régulière de $7 \%$ par an depuis 1950 en dépit des contrariétés conjoncturelles qui peuvent crisper les marchés ${ }^{5}$.

Les directions des flux se diversifient : à la logique des flux Sud-Nord à sens unique se surimposent désormais des déplacements Nord-Nord et Nord-Sud qui caractérisent ce «nouvel âge » des migrations (Castles and Miller 1993) dans un contexte croissant d'internationalisation du marché du travail. L'essor croissant du tourisme met en valeur ces disparités tout en jouant un rôle grandissant dans la redistribution des revenus au profit de nouvelles zones de production. Cette nouvelle réalité, dans une conjoncture de contrôle des flux migratoires, explique la prégnance des corrélations qui s'établissent entre tourisme et migration.

Contrôle des frontières et projets touristiques

23 Face aux politiques de régulation des flux et de fermeture des frontières entre pays le plus développés et pays les moins développés, les plus démunis n'ont plus le droit de quitter leur terre où les perspectives d'avenir sont rares. Les clandestins tentent de "prendre d'assaut la forteresse Europe $»^{6}$, les nouveaux boat-people ne parviennent pas à rejoindre l'Australie intransigeante ${ }^{7}$.

Guengant (1996: 114) expose les nouveaux paradigmes des migrations internationales et du développement qui considèrent le développement comme « seul moyen susceptible d'éviter à terme les migrations internationales. L'argumentation est la suivante: les écarts de revenus entre les pays de départ et d'arrivée constituant la cause principale des migrations internationales, la diminution, puis la suppression de ces écarts par convergence des niveaux de revenus au cours du processus de développement rendra caduc à terme le besoin d'émigrer ". Dans ce contexte sont entrepris de nouveaux projets de développement mettant particulièrement l'accent sur les «potentialités » touristiques dans les pays du sud : la création d'un lieu touristique autoriserait alors des transferts de capitaux et de consommateurs dans des pays traditionnellement de départ.

Sur le plan théorique, le tourisme apporte une solution dans le cadre de ces programmes de développement dans les pays du sud, mais c'est sans compter avec les logiques des touristes, leurs choix et les représentations de ces régions, la difficulté de créer une nouvelle destination sur un marché très concurrentiel. De la même manière, ces projets ne prennent pas en compte les motivations profondes et complexes des migrants potentiels où «contrairement à une idée reçue largement admise, (...) la pauvreté 
(ou plus généralement le sous-développement) n'est pas véritablement le facteur principal, fondamental des migrations internationales » (Guengant, 1998, p. 18).

Tourisme et réalités économiques

Vers les lieux touristiques ${ }^{8}$, miroirs de la mondialisation, convergent des hommes aux motivations diversifiées et aux logiques de déplacements contrastées (matérialisées par l'avion, le taxi collectif, ou le vélo), à l'image d'un «monde qui bouge à plusieurs vitesses ». Les arrivées de touristes internationaux avoisinent 700 millions en 2000 (ou 435 millions avec une définition plus rigoureuse) pour des recettes internationales de 475,9 milliards de dollars US. Les voyages inter-continentaux représentent moins de $25 \%$ des déplacements, les flux privilégient les périphéries des régions les plus développées et les plus anciennement touristiques qui concentrent l'essentiel des recettes $^{9}$. Si la domination des principaux pays touristiques demeure, de nouvelles destinations s'affirment: plus de soixante-dix États ont reçu plus d'un million de touristes internationaux en $1999^{10}$ (O.M.T., 2000).

Sans remettre en cause la position des régions dominantes, la croissance du marché constitue un enjeu économique fondamental pour de nombreux prétendants. Les perspectives offertes et imaginées attirent les migrants d'autant plus facilement que les schémas migratoires traditionnels sont remis en cause par la fermeture des frontières vers les foyers économiques du Nord. L'impact est incontestable dans certains pays comme la Chine où le visiteur dépense en moyenne $520 \$$ US par séjour ${ }^{11}$, le Mexique ou la Malaysia ${ }^{12}$. Les enjeux sont essentiels pour les petits États qui jouent pleinement cette carte tels la République Dominicaine et Porto Rico avec des recettes de l'ordre de 2,5 à 3 milliards de dollars US et des progressions annuelles de 15 à $20 \%$. Dans les îles touristiques, le poids des recettes du tourisme peut facilement couvrir 50 à $80 \%$ du $\mathrm{PNB}^{13}$. De nouveaux marchés se structurent dans ces pays émergents: les investissements semblent faciles, des emplois sont créés, l'argent circule, de nouvelles migrations internes et internationales s'organisent.

Anciennement développés ou émergents, les lieux touristiques constituent d'intéressants laboratoires pour analyser les logiques des mobilités contemporaines et leurs recompositions multiformes. Ils occupent des positions centrales; point de rencontre entre des flux de nature différente (touristiques et migratoires) où se dessinent des interrelations, des chevauchements, des filiations.

Le marché touristique : nature et logiques

29 L'importance du marché touristique n'est plus à démontrer: il est l'un des cinq premiers secteurs d'exportation (grâce aux recettes touristiques) pour $83 \%$ des États et la principale source de devises pour $38 \%$ d'entre eux. Les programmes de restructuration économique conduits sous l'égide du Fonds Monétaire International et de la Banque mondiale, qui mettent l'accent sur l'indispensable ouverture des États sur la mondialisation et sur le renouvellement des sources de revenu pour sortir de la dépendance envers les exportations traditionnelles (comme le cacao, les bois tropicaux ou les minerais). Ils insistent sur les perspectives offertes par le tourisme comme l'explique Konadu-Agyemang (2001) à travers l'exemple de Ghana.

Les capitaux internationaux régissent le secteur touristique. Localement des petits investisseurs impulsent des projets, des travailleurs expatriés y investissent parfois leurs économies mais les logiques de création d'un lieu touristique s'érigent aux échelles supérieures, impulsées par des entreprises multinationales, spécialisées, qui 
choisissent leurs lieux d'accueil. Le fonctionnement du lieu s'articule alors entre les logiques du marché local et les règles du marché global.

Marché global et fonctionnement local

31 Le fonctionnement du marché repose sur une contradiction nécessaire entre la concentration croissante du capital entre les mains de quelques entreprises multinationales et à l'autre extrémité, un foisonnement d'entreprises et de petites initiatives privées. Les unes, en position intermédiaire, sous-traitent avec les entreprises internationales et assurent l'encadrement de l'activité à l'échelle du territoire d'accueil. Les autres, à la base, sont des petites entreprises, marginales sur le marché international, et dont les résultats dépendent des choix et des politiques des multinationales (Britton, 1989).

Cette économie duale (Dahles, 1999) est dominée par des groupes multinationaux au capital transnational et mondialisé comme dans le domaine des transports aériens et de l'hôtellerie où les stratégies d'alliance, d'union ou de rachat permettent de faire des économies significatives en réduisant la concurrence (Youell, 1998; Agarwal and alii., 2000) à l'image du marché britannique où les cinq premiers voyagistes détiennent $72 \%$ des parts du marché en 1991 contre 49,5\% en 1983 (O’Brien, 1998). Ces formes de concentration dans la commercialisation des séjours, des titres de transports s'inscrivent dans la logique des marchés ${ }^{14}$. Les gouvernements qui veulent attirer ces consommations touristiques et développer ce secteur d'emploi traitent avec ces entreprises internationales.

Les voyagistes sous-traitent localement les activités qu'ils organisent, depuis la vente de services jusqu'à l'approvisionnement alimentaire, en faisant jouer la concurrence entre de nombreuses petites entreprises interdépendantes. Ces méthodes sont identiques à celles des autres entreprises multinationales qui n'hésitent pas à délocaliser selon les opportunités. Notons qu'ici l'emploi local est véritablement ancré dans son territoire d'origine : on ne peut pas délocaliser la plage ni la chambre avec vue sur la plage. Certes, les coûts de gestion pourront être réduits au minimum sur place mais il reste une partie non transférable. En fait, dans ces destinations émergentes où la main-d'œuvre est bon marché et abondante, les petits emplois fourmillent et les avancées technologiques sont assez lentes.

Des investissements assez modestes peuvent rapidement permettre la création d'emplois tant formels qu'informels dans le secteur touristique. Selon les statistiques publiées par l'OCDE, la création d'un emploi dans le secteur touristique demande un investissement sept fois moins important que celle d'un emploi dans l'industrie (Montanari, 1995 : 61). «Le fort potentiel de création d'emplois par le tourisme est l'une des raisons pour lesquelles les gouvernements sont intéressés par promouvoir son développement » (Smith, 1997).

Dans les pays méditerranéens, quelques trois millions de personnes travaillent ainsi dans l'hôtellerie, dont 70 \% dans la partie européenne selon un ratio d'un emploi pour cinq lits de classe moyennes (Sessa, 1983). Les analyses de Renucci (1990) montrent que trois à cinq lits hôteliers procurent un emploi direct dans l'établissement et deux à huit emplois dans le reste du secteur touristique. Le nombre d'emplois serait donc de l'ordre de dix millions dans les régions méditerranéennes (Montanari, 1995). Berriane (1983) relève même un taux de 0,7 emploi par chambre à Agadir, taux qui peut descendre à « 0,42 emploi par chambre d'hôtel » dans l'hôtellerie haut de gamme (Huetz de Lemps, 1989 ; Singaravelou, $\left.1989^{15}\right)$. 
Les caractéristiques du marché local de l'emploi

Les services sont sur-représentés, mobilisant couramment "plus des trois-quarts des actifs, cette proportion pouvant s'élever jusqu'à près de $90 \%$ pour certaines stations » (Dewailly et Flament, 1993). Deux catégories d'emplois se distinguent à l'échelle locale : ceux en relation directe avec les entreprises touristiques internationales ou nationales, qui gèrent les flux et l'accueil, et une multitude d'emplois plus ou moins formels qui se greffent sur ce noyau central.

Hier comme aujourd'hui, le marché touristique attire de la main-d'œuvre aux compétences inégales. L'espace d'accueil est rapidement débordé par une demande " inhabituelle ", en décalage avec les activités traditionnelles. L'arrivée de travailleurs répond à un double déficit local : quantitatif (nombre d'actifs disponibles insuffisants pour répondre à la demande en pleine saison) et qualitatif (personnel qualifié, notamment pour les fonctions décisionnelles qui demandent des compétences spécifiques). Le déficit peut être compensé par l'existence de formations intermédiaires plus accessibles, mais les investissements demandés sont freinés par les perspectives professionnelles aléatoires, notamment avec l'importance des contrats de courte durée ${ }^{16}$. L'émergence du marché touristique suscite donc des migrations de proximité, à partir de l'hinterland rural, et aussi de migrations relevant d'un plus vaste espace régional comme cela a été souligné dans la région de l'Algave (Cavaco, 1980) et dans les îles Baléares où les ressortissants nord-africains remplacent désormais les premiers migrants originaires du sud de l'Espagne (article de Salvà Tomàs dans ce dossier).

L'activité touristique crée un nouveau marché, qui se caractérise par une nouvelle hiérarchisation du monde du travail où la grille des responsabilités et des salaires, établie suivant des niveaux de qualifications, entraîne des catégories de migrations spécifiques.

39 - Au sommet de l'édifice, des investisseurs étrangers ou nationaux, peu présents sur le site ; ces décideurs appartiennent à des élites internationales très mobiles. - Localement, les gestionnaires, personnel très qualifié, sont extérieurs à la région, comme l'essentiel des employés spécialisés (du cuisinier au professeur de golf) parmi lesquels sont recensés quelques nationaux. Ces actifs au cœur du marché local ne restent en général que quelques années dans un même lieu touristique. Ils ont leur propre circulation migratoire dont la progression se fait à mesure de la capitalisation des expériences, commençant par des petites structures périphériques pour aller vers les lieux les plus réputés, où le niveau de compétences exigé autorisent de meilleures rémunérations. Ce parcours peut s'effectuer dans le cadre d'un même groupe ou avec des changements successifs d'employeurs suivant des stratégies individuelles. À terme, l'employé peut aussi choisir de devenir entrepreneur ${ }^{17}$.

41 - De nombreux emplois sans qualification spécifique, demandant de l'habilité et du sens du relationnel, sont occupés par de jeunes originaires des métropoles proches; ils disposent d'une formation de base, parfois dans le tourisme et de quelques rudiments en langue étrangère.

42 - Enfin les emplois, sans compétence particulière, interchangeables et compressibles, sont le lot de la population d'origine et de migrants de proximité.

43 Parallèlement aux emplois directement régis par les structures centrales, d'intéressantes stratégies professionnelles sont définies dans le cadre de petites affaires privées; émigrés de retour au pays, émigrés venant d'espaces voisins et petits 
entrepreneurs locaux se retrouvent sur les périphéries du marché local pour élaborer des services et autres produits spécifiques qui enrichissent l'offre en mettant l'accent sur le particularisme local.

Flexibilité et migration

Le marché touristique s'accorde assez facilement avec le nouveau contexte migratoire caractérisé par "des migrations à durée et itinéraire variables, plus "flexibles". Guengant (1996: 110) constate que "cette flexibilité des migrations va de pair avec la recherche par les entreprises du secteur formel, au Nord comme au Sud, d'une plus grande flexibilité de la main d'œuvre, et des processus de production ». Le tourisme est certainement le secteur d'activité qui utilise la flexibilité avec le plus de facilité, gérant au plus juste les besoins en main-d'œuvre dans un secteur en cours de structuration avec des capitaux volatiles.

Le volume d'employés permanents, qui peut varier du simple au double pour une même tâche, représente un cinquième à un dixième de l'effectif maximum présent pendant la pleine saison. Cela dépend du coût local de la main-d'œuvre, de l'existence d'une basse saison autorisant un minimum d'activité. Les demandes variables et changeantes sont inégalement réparties sur l'ensemble d'une année comme au cours de la saison ou même de la journée. En raison de la nature de la demande et de la production, la flexibilité est la règle (Williams and Montanari, 1995), renforcée par des aléas politiques et économiques ${ }^{18}$.

Les clivages sociaux, ethniques, de genre, d'âge, de régularité sont abondants sur le marché du tourisme. La part d'emplois dits «féminins " est importante, notamment pour les activités à temps partiel. Dans le secteur de l'accueil au Royaume-Uni, les femmes, largement concentrées dans les fonctions de ménage et de réception, n'occupent que $4 \%$ des fonctions intermédiaires et $1 \%$ des postes de direction (Jordan, 1997).

Bien sûr des groupes ethniques se sont spécialisés dans certains types de restaurations à l'étranger à l'image des restaurants indiens ou chinois, des pizzerias et autres "sandwicheries" mais le phénomène déborde ici largement les seuls commerces spécialisés pour mettre en évidence l'un des secteurs de prédilection des travailleurs émigrés. King (1995) observe que dans les pays d'immigration plus récente, les travailleurs étrangers avec un permis en règle sont encore plus présents dans les secteurs des services et dominent dans celui du tourisme comme en Espagne où $73 \%$ d'entre eux travaillent dans les services en 1990 pour $15 \%$ seulement dans l'industrie, 7 \% dans le bâtiment et $4 \%$ dans l'agriculture (Charmes et alii.,1993).

La part importante du travail au noir (qui peut représenter un tiers de l'activité) intègre facilement des travailleurs clandestins telles des femmes de chambres philippines en Italie et en Espagne ou encore des employés de cuisine marocains ou indiens. Des spécialisations se dessinent. De nombreux emplois ne nécessitant que de faibles compétences pour des tâches universelles expliquent l'ouverture permanente aux immigrants dans les pays touristiques depuis longtemps. Ces emplois faiblement rémunérés intéressent peu les populations nationales : les salaires sont réduits pour maintenir des prix compétitifs. Toute une économie souterraine s'organise autour de ces tâches flexibles qui satisfont des migrants de passage, "faux-touristes » à la recherche d'un emploi saisonnier. En dépit de la régulation des flux aux frontières, dans nos régions touristiques, des secteurs d'activité ont besoin des travailleurs émigrés. L'absence de reconnaissance officielle les contraint à vivre dans la 
clandestinité et la précarité, dans la crainte de la police ou de la parole non tenue d'un employeur. Ces emplois instables, dans l'ombre, qui constituent la face cachée de l'entreprise, sont indispensables à son fonctionnement actuel.

Un marché local renouvelé

Le renouvellement de l'économie locale se traduit en terme d'emplois directs, indirects et induits. Une redistribution de la part des revenus touristiques laissée localement s'effectue dans l'espace d'accueil et à travers la société. Suivant leur degré d'implication dans l'activité touristique, trois catégories peuvent être distinguées : - Les emplois directs dans la logistique des transports et agences de voyages, l'hôtellerie, la restauration et dans un large secteur de services (sports, animation...). Le nombre d'employés est réévalué dans les administrations et des commerces spécialisés se greffent sur le marché. Une foule de petits entrepreneurs gravitent autour des centres d'accueil (guides, chauffeurs, vendeurs ambulants).

51 - Les emplois indirects soutiennent le système touristique. Ils concernent toutes les tâches d'approvisionnement, de construction et de maintenance des infrastructures. - Les emplois induits résultent du renchérissement de l'économie locale. La circulation de l'argent introduit par les touristes stimule l'économie locale. De nouvelles demandes, désormais solvables, contribuent à la diversification de l'offre de services en constituant une nouvelle clientèle.

La "mise en tourisme » des lieux s'accompagne d'un bouleversement des structures traditionnelles en introduisant une nouvelle logique qui perturbe les populations plus âgées dans l'impossibilité de revoir leur système de production, de transformer leur rythme de vie. L'épreuve peut être difficile pour certains habitants qui seront " contraints " à changer de cadre de vie, s'installant en périphérie ou émigrant vers l'intérieur des terres. En ce sens, la construction d'un nouveau lieu touristique peut être à l'origine de flux de départ. Le tourisme introduit une rupture, parfois brutale. La mise en tourisme du lieu s'accompagne d'une modernisation et d'un désenclavement au plan international vis à vis de l'international (des dessertes aériennes) mais aussi national (avec la réalisation de voies routières à grande vitesse reliées aux centres vitaux du pays). Cette double accessibilité, indispensable pour drainer les visiteurs, facilite les flux de migrants potentiels.

54 Toute l'économie locale est stimulée par la présence de ces nouvelles clientèles. Des commerces deviennent polyvalents, partagés entre les demandes des visiteurs et celles des populations locales, des services parfois informels s'organisent. Après une première période de remise en cause des fondements traditionnels de l'espace et de la société d'accueil, le tourisme amène une restructuration de l'économie locale.

Une foule de petits entrepreneurs

Le petit entreprenariat est particulièrement présent dans les régions nouvellement touristiques. Un secteur informel se développe, plus ou moins aisément, selon les positions des pouvoirs politiques. Certains comptoirs touristiques s'affichant comme des destinations de luxe excluent ces initiatives, seuls sont présents les entrepreneurs sous-traitant officiellement avec les grands groupes. On peut ainsi opposer Nusa Dua à Kuta sur l'île de Bali; cette dernière station concentre une multitude de petits entrepreneurs dans les domaines du commerce, de l'art ou des transports. Les fameux becak ou « vélo-porteurs » qui confortent cette impression d'exotisme authentique chez le visiteur sont à l'image des stratégies professionnelles des acteurs nouvellement 
arrivés. Si dans les métropoles, les emplois de becak attirent des populations parmi les plus démunies, les profils ne sont pas les même dans les stations. Outre la qualité du matériel, les porteurs sont de jeunes hommes connaissant quelques mots d'anglais et venant des grandes villes. Dans ces stations ils obtiennent des revenus les plaçant au niveau des classes moyennes nationales. Leur travail n'a rien à voir celles des becak urbains, surchargés, corvéables à merci ; ici, un seul passager par vélo et les bagages encombrant font l'objet de surtaxe. Pour moins d'un kilomètre sur une promenade littorale, la course sera payée dix à cinquante fois plus que dans la capitale aux conditions de circulation beaucoup plus éprouvantes. Les becak travaillent avec les boutiques d'artisanat et les hôteliers comme à Yogyakarta (Van Gemert and alii., 1999), conduisant les voyageurs de l'arrêt de bus «au meilleur hôtel de la station » en lui vantant ses prix et sa qualité au détriment des autres concurrents. Des liens sociaux s'établissent autour du tourisme dans la communauté locale. Des réseaux se forment, on se renvoie les clients, à chacun son domaine. Rien n'est formalisé, le touriste va d'une adresse à l'autre, parfois accompagné pour ne pas le laisser s'égarer vers des concurrents.

Un fonctionnement cohérent s'installe rapidement au sein de cette activité très fragmentée qui associe des travailleurs venant d'horizons divers. La société locale renouvelée fait preuve d'une culture d'innovation. Toutes les demandes potentielles des touristes trouvent localement des réponses.

Sur la base du découpage proposé par Dahles (1999b), ces multiples initiatives individuelles peuvent être regroupes en huit catégories :

58 - en premier lieu, le secteur de l'hébergement, des pensions de famille aux locations de petites dépendances réaménagées ;

59 - puis celui de la restauration et des débits de boisson pouvant se réduire à la vente ambulante ;

60 - viennent ensuite les emplois dans le domaine des transports ;

61 - des services d'accompagnement, d'encadrement des touristes (comme les guides avec ou sans statut officiel);

62 - les commerces de vêtements, bijoux, artisanats et objets d'art, dans le cadre de boutiques ou de la vente ambulante ;

63 - des locations en tout genre : vélos, mobylettes, ombrelles, équipement de plongée ou simple canne à pêche ;

64 - des services de beauté (massage, manucure, coiffure de mode ou exotique, maquillage);

65 - des services de divertissement, du spectacle, de l'amusement (musique, théâtre, danse).

66 Ces petits entrepreneurs largement issus de l'immigration jouent un rôle essentiel dans le fonctionnement de la station. Parallèlement aux emplois inscrits dans le cadre des structures officielles, les occasions sont réelles. Bien sûr à côté des entreprises respectables se développe une part plus ou moins importante d'activités pouvant relever de commerce illicite et illégal (Crang, 1997), en lien avec la prostitution, les jeux clandestins et la drogue.

67 Le tourisme est générateur de migration de travail tant au niveau local, régional qu'international. Dans les régions touristiques les plus développées, comme la Côte 
d'Azur, la Costa del Sol, on note même désormais une inversion des mouvements migratoires (avec l'arrivée d'actifs venus du Nord de l'Europe dans ces régions longtemps émettrices de migrants). Et, même lorsque la part des emplois occupés par des résidents est importante, des flux saisonniers continuent de converger vers ces marchés.

Des liens étroits existent entre les logiques du marché du tourisme et les migrations de travail, et au-delà, la complexification progressive des mouvements migratoires et touristiques, à l'échelle mondiale, et leur convergence au profit de lieux «à dominante " touristiques nous interroge sur le continuum tourisme/migration dans le cadre d'un système global de mobilités.

Interrelations tourisme-migration. études de cas

Des interrelations croissantes à partir des lieux touristiques, entre les mobilités autorisées et encouragées (déplacements de loisirs) et celles plus ou moins tolérées (migrations), laissent apparaitre plusieurs scénarios entre des migrations spontanées, des touristes nouveaux résidents et des "faux touristes" contournant les réglementations pour émigrer. Les différents scénarios peuvent être présentés à travers cinq cas de figure.

Cas 1 - Tourisme et marché : un appel de main-d'œuvre

70 La création d'un marché touristique entraîne un premier appel de main d'œuvre (Kontogeogopoulos, 1998) avec un emboîtement des échelles migratoires et de multiples logiques individuelles. Son fonctionnement se fonde sur un double accueil : des visiteurs en vacances (une demande de services pour une consommation locale) et des nouveaux actifs attirés par leur présence.

71 Dans ce processus caractérisé par l'arrivée de flux touristiques réguliers, deux phases migratoires distinctes apparaissent.

72 - Dans un premier temps, la structuration du marché est soutenue par l'arrivée d'une première génération de migrants. Au cours de cette période, des populations locales peuvent se déplacer, s'installer en périphérie ou émigrer.

73 - Dans un second temps, le marché établi reste fortement lié aux migrations en raison de l'important renouvellement du personnel spécialisé et de la nature de certaines tâches, incompressibles, peu rémunérées remplies par des travailleurs émigrés au cours de saisons plus ou moins courtes.

74 Ces flux sont particulièrement significatifs dans des espaces insulaires, comme les îles Hawaii marqués par une modernisation et un enrichissement accélérés (Minerbi, 1994). L'archipel accueille quelques sept millions de touristes et compte environ 100000 lits touristiques contre cinq hôtels et six pensions de famille au milieu du siècle dernier. Le renouvellement $\mathrm{du}$ peuplement par vagues migratoires successives est important ${ }^{19}$ (Kim, 1994). La croissance réelle - le budget moyen d'une famille de quatre personnes est supérieur de $37,7 \%$ à la moyenne nationale - s'accompagne d'inégalités contraignant certains habitants des îles à émigrer vers le continent tandis qu'arrivent de nouvelles vagues d'immigrants ${ }^{20}$ (Minerbi, 1994). La migration "est à la fois un catalyseur et une conséquence d'un changement social et économique» (Connell, 1987) et aucun société touchée par le tourisme ne peut alors rester sans réaction.

Cas 2 - Tourisme et retour des émigrés

Dès les années soixante et soixante-dix, les études conduites dans les régions méridionales de l'Europe ont permis de mettre en évidence les filiations qui 
s'établissaient entre les retours des émigrés et les investissements réalisés localement dans le tourisme, comme dans le sud de l'Italie avec l'exemple de la ville touristique d'Amantea (King, 1984), sur la côte dalmate et dans ses îles (Baucic, cité par King, 1995 ; Bennett, 1979), au Portugal (Cavaco, 1993), sur l'ensemble du littoral méditerranéen (Williams, 1993a). A partir de l'exemple de Nazaré, Mendonza a montré comment les migrants ont privilégié des investissements immobiliers en relation avec le marché touristique émergent (Mendoza, 1982), des études similaires sont aujourd'hui conduites à partir des stations balnéaires marocaines.

76 Les atouts de ces expatriés de retour sont de plusieurs ordres. Outre les capitaux accumulés et disponibles, ils reviennent avec une certaine connaissance des régions émettrices de touristes: les pratiques récréatives, les types de consommations et certaines langues étrangères leur sont familières. Trois perspectives de travail se présentent à leur retour comme l'expose Ardittis (cité par King, 1995) :

77 - un retour à la terre, mais la tâche est dure pour des revenus faibles ;

78 - la recherche de fonctions meilleures et plus valorisantes sur le plan social : le secteur tertiaire est tout désigné mais les emplois publics sont rares ;

79 - il reste alors l'initiative individuelle dans le secteur privé et notamment dans les commerces et services en relation avec le tourisme.

80 Il est à noter que les pratiques des émigrés de la seconde génération s'alignent davantage sur celle des touristes (Cavaco, 1993). A la différence de leurs aînés qui privilégiaient les relations avec leur village d'origine, les plus jeunes séjournent davantage dans les stations balnéaires parmi les touristes nationaux et internationaux. Cas 3 - Tourisme, multi-résidence et transfert d'activité

81 Dans les lieux touristiques, la présence des visiteurs est davantage étalée dans le temps et les séjours sont plus fréquents; parmi les plus mobiles, des retraités libres de leur temps, des touristes qui transfèrent partiellement leurs activités ou qui décident d'émigrer dans ces lieux.

82 Ces touristes plus mobiles, séjournant à l'étranger plus longtemps et susceptibles de partagés leur vie entre plusieurs lieux proviennent de quelques foyers émetteurs, essentiellement européens : la Rhénanie du Nord-Westhalie, la Bavière et le BadeWurtemberg, le Sud-Est et le nord de l'Angleterre, les métropoles du Randstad (Amsterdam, Rotterdam, La Haye), les régions urbaines de Vienne, Stockholm ou Paris. Les métropoles nord-américaines et japonaises ont également leurs bassins privilégiés sur les côtés caraïbes et sur les pourtours méridionaux de la mer de Chine. Les nouvelles mobilités où se mêlent tourisme, double résidence et transfert d'activité se font surtout dans un cadre régional.

83 Ces formes complexes de mobilité s'inscrivent dans un continuum entre déplacements touristiques, mobilité résidentielle et transferts d'activités. Ces nouvelles situations peuvent s'expliquer partiellement par l'évolution du monde du travail où les personnes les plus qualifiées connaissent une plus grande mobilité qui entraîne une plus grande dispersion géographique des parents et amis (Williams and Hall, 2000). L'accumulation d'expériences de vie dans des espaces différents facilite ces changements de résidences lors de la retraite.

84 Les lieux touristiques les plus concernés appartiennent aux régions littorales méditerranéennes marquées par l'émigration jusqu'au début des années 1980 ; elles connaissent une inversion des flux (Montanari and Cortese, 1993). Des Nord-européens 
s'installent aux Baléares (Duhamel, 1997 ; Salvà-Tomàs, 1991), sur les côtes espagnoles, en Toscane et se diffusent dans les arrière-pays méridionaux introduisant une gentryfication de certains lieux (Phillips, 1993). Ces flux concernent aussi la rive sud de la Méditerranée avec l'achat de maisons traditionnelles dans les médinas de Marrakech, d'Essaouira ou d'Alexandrie.

Les mobilités des retraités

85 Particulièrement fréquents dans les régions méditerranéennes, ces déplacements connaissent une situation favorable dans les pays les plus développés (entre allongement de la durée de vie et pouvoir d'achat conséquent). A.M. Warnes (1994) établit une filiation entre les lieux connus et pratiqués par les touristes et le choix d'un nouveau lieu de résidence pour la retraite. Ainsi les ressortissants anglais qui s'établissent dans les provinces de Malaga, d'Alicante ou des Baléares. Après s'être familiarisé avec ces lieux, la cessation d'activité peut être l'occasion d'un nouveau projet de vie privilégiant des périphéries ensoleillées. Parmi les retraités, selon O'Reilly (1995), nous pouvons distinguer cinq catégories :

86 - les expatriés sont des résidents permanents, ils s'identifient à leur région d'accueil, y créent de nouvelles sédentarités. Parfois propriétaires, ils n'ont plus l'intention de rentrer dans leur pays d'origine ;

87 - les résidents, avec un statut légal de résident, retournent régulièrement dans leur pays d'origine mais pour des séjours de deux à cinq mois. Ils y rencontrent des parents et amis et peuvent y posséder une résidence secondaire ;

88 - les visiteurs saisonniers restent attachés à leur pays d'origine dans lequel ils maintiennent leur domicile principal. Ils effectuent régulièrement des séjours de deux à six mois ;

89 - les visiteurs occasionnels, propriétaires d'une résidence secondaire, n'y font que des séjours irréguliers ;

90 - enfin les retraités touristes. Ils se déplacent fréquemment mais séjournent dans les zones touristiques en louant les hébergements nécessaires pour leurs séjours de courte durée.

91 D'après $\mathrm{A}$. Williams ${ }^{21}$, «Près de 72000 pensions de retraite seraient payées par les institutions britanniques dans les pays méditerranéens (Espagne, Italie, France, Chypre, Portugal, Malte, Grèce) en janvier 1995, avec un total en progression moyenne de 30-35 \% depuis 1990 ». La Costa del Sol est la première destination pour les Britanniques (Williams and alii, 2000) avec un contingent de 16700 retraités sur un total de 50000 résidents au milieu des années 1995, 2000 sont installés en Toscane (sur 4000 résidents), 1700 à Malte (sur 5000 ) et 1600 dans l'Algarve portugais (qui compterait plus de 50000 Britanniques séjournant plus de trois mois, dont 10000 résidents permanents).

92 Ces mouvements sont également établis entre les métropoles nord-américaines d'une part, la Sun Belt et certaines îles des Caraïbes de l'autre. Ils se dessinent aussi depuis le Japon et la Corée du Sud en direction des côtes thaïlandaises, des îles des Mers du Sud et des côtes australiennes

Du tourisme au transfert d'activité

93 Les séjours à des fins récréatives peuvent entraîner un transfert partiel ou complet d'activité. Cet itinéraire de vie est particulièrement développé dans les régions de fortes fréquentations touristiques comme dans les destinations émergentes. Eaton 
(1995) ou Madden (1999) ont analysé le rôle des migrants britanniques ou irlandais qui investissent sur la Costa del Sol, dans le secteur de la restauration et des bars en relation avec des clientèles essentiellement britanniques. Il apparaît que les choix reposent d'abord sur des raisons sociales et sur la recherche d'une autre qualité de vie, les stratégies purement financières ne concernant qu'une faible proportion (seulement $8 \%$ selon les enquêtes réalisées par Madden).

Ces touristes qui choisissent de changer de cadre de vie durablement élaborent des projets variés allant du transfert, complet ou partiel, d'une activé qui demeure inchangée à la définition d'une nouvelle activité rémunératrice :

- les transferts les plus aisés correspondent aux professions libérales qui peuvent être conduites à partir de plusieurs lieux, sans lien avec l'activité touristique ;

96 - d'autres transferts se font en relation directe avec le marché touristique et sans changement dans la nature de l'activité. Par exemple un restaurateur décide d'ouvrir un nouvel établissement dans une station, et ainsi changer de cadre de vie sans changer de métier ;

97 - le désir de changer de cadre de vie peut aussi nécessiter un changement d'activité. Le migrant s'oriente alors vers les locations touristiques, le commerce, l'artisanat. Les investissements de départs peuvent être modestes, l'expérience relève avant tout d'une volonté et de stratégies personnelles.

98 Contrairement aux travailleurs émigrés qui peuvent partir vers l'inconnu, pour ces nouveaux migrants, la connaissance préalable des lieux est importante. Ces nouveaux mouvements s'inscrivent dans le contexte des sociétés d'Europe occidentale qui "produisent suffisamment de rentiers et d'actifs pouvant se permettre de relocaliser leur activité professionnelle» (Knafou, 1997). La recherche d'une qualité de vie, d'un équilibre personnel entre ces lieux de vie multiples prime sur les motivations strictement matérielles, la diversité des logiques est réelle: si l'héliotropisme domine, la quête d'espaces et la qualité de l'environnement sont déterminants pour d'autres comme l'illustre Müller avec ces ressortissants allemands installés dans le Norden (article de Müller dans ce dossier).

Cas 4 - Tourisme et diaspora

Des relations peuvent être établies entre les logiques des flux touristiques et les territoires migratoires. Peu de communautés sont concernées de façon significative par ces interactions tourisme-diaspora. Il faut que le niveau de vie global du pays d'origine soit suffisamment élevé pour qu'il puisse dégager une masse critique de touristes séjournant à l'étranger et qu'il existe au préalable une communauté structurée à l'étranger.

100 Les logiques des mouvements internationaux des Sud-coréens entrent tout à fait dans ce cas de figure (Kang and Page, 2000). Des processus similaires se mettent en place au sein des communautés chinoises en liaison avec l'augmentation progressive du niveau de vie. Mais les déplacements associent encore largement les visites à des proches et les pratiques récréatives avec des échanges de service non payants. Or dans le cas de Sudcoréens il s'agit bien de pratiques touristiques donnant lieux à des services marchands et privilégiant les ressortissants de la communauté lors des voyages à l'étranger. Cela existe au sien d'autres communautés mais sans atteindre le niveau significatif des Coréens. 
101 Les Sud-coréens sont plus de quatre millions à faire des expériences touristiques à l'étranger et ces voyages n'ont commencé à se développer que depuis la fin des années quatre-vingts. Pour organiser leurs séjours, ils privilégient les services d'agences nationales travaillant avec des ressortissants établis dans les pays et les villes visitées. Les membres de la communauté à l'étranger commercialisent des chambres chez l'habitant, tiennent des restaurants coréens et les étudiants s'improvisent guides en fonction des demandes. Les spécificités de ce fonctionnement communautaire sont favorisées par l'absence de culture touristique et le fort degré d'altérité auquel sont confrontés les ressortissants qui voyagent à l'étranger (la limite la plus évidente venant des difficultés linguistiques).

L'offre touristique des membres de la communauté à l'étranger est exclusivement orientée vers les nationaux (via les agences et des annonces rédigées en coréen). Les services sont payants et les Coréens sont réputés pour leurs généreuses dépenses lors de leurs voyages, dans certaines situations, ils paieront même plus cher un service qui aura la garantie du lien communautaire, comme l'accueil dans un aéroport par un taxi occasionnel travaillant avec la structure d'hébergement réservée. Ces réseaux n'ont pas besoin d'être déclarée dans le pays d'accueil, leur clientèle est captée à partir du pays d'origine (à l'exception des restaurants qui ont une double clientèle).

103 L'association d'activités officielles dans le pays d'accueil (étudiants, artistes, commerçants) permet de s'adapter à la flexibilité demandée. Et comme tout système touristique où il $\mathrm{y}$ a des tâches simples à effectuer, notamment pour l'entretien des chambres chez l'habitant, la communauté emploie ses clandestins, appartenant aux minorités coréennes venant de Chine (région du Yambian), dont les emplois sont partagés entre trois ou quatre structures d'accueil différentes.

L'existence de ces économies parallèles entre ressortissants d'un même pays, dans un contexte de croissance du marché touristique, encourage des nouveaux projets migratoires. Les ressources financières générées par les touristes coréens à l'étranger constituent une source de revenus appréciables pour la communauté en exil. Un rapprochement peut même être envisagé à l'échelle mondiale entre les lieux touristiques fréquentés par les Sud-coréens et l'existence d'une communauté d'émigrés. Durant les dix dernières années les émigrés les plus anciens sont rejoints par de nouveaux migrants, aux statuts variables (résident, profession libérale, étudiant) selon les difficultés administratives. Sont privilégiées certaines métropoles nordaméricaines et européennes (Londres, Paris, Toronto, Vancouver, Los Angeles...) ainsi que des destinations touristiques réputées en Asie du Sud-Est, dans le Pacifique (Guam, Hawaii) et sur les côtes australiennes et néo-zélandaises. Plus l'altérité est importante et plus les liens communautaires sont recherchés, comme dans le cadre des déplacements.

105 Dans le cas des Sud-coréens, il ne s'agit plus seulement des facilités professionnelles qui peuvent être développées au contact de compatriotes - comme pour les entrepreneurs britanniques sur la Costa del Sol - mais bien d'une logique de diaspora avec le développement de services spécifiques souvent indéchiffrables pour les autres touristes. Une territorialisation se construit à travers ces réseaux alimentés par des mobilités multiformes (tourisme, transfert d'activité, migration) des membres d'une même communauté. Entre pratiques touristiques, choix de nouveaux lieux de vie et liens avec le pays d'origine, les Sud-coréens se construisent une "réticularisation territoriale » (Lévy, 2000). 
Cas 5 - Le tourisme prétexte migratoire de tête de pont à partir desquels s'organisent de nouveaux flux migratoires. Certaines filiations peuvent être établies entre des pratiques touristiques et des flux migratoires réversibles traduisant un transfert d'activités au profit des foyers émetteurs de touristes, comme l'illustrent des études conduites sur le marché de la prostitution japonais (Muroi and Sasaki, 1997).

112 Dans le cas du Japon, ce sont les transformations progressives de pratiques des touristes qui ont accéléré ce glissement d'activité. Les premières générations de touristes japonais, des années soixante à la fin des années soixante-dix, étaient essentiellement composées d'hommes voyageant en groupe - on parlait volontiers de voyages d'affaires pour lesquels les entreprises louaient les services de voyagistes 
spécialisés. Les destinations privilégiées était les pays voisins - la Corée du $\operatorname{Sud}^{23}$ et Taïwan qui n'étaient pas encore les NPI - puis la Thaillande et les Philippines, dans le contexte de la guerre froide avec la présence de nombreuses bases américaines et un marché de la prostitution en plein essor. A partir des années quatre-vingts, ces voyages "d'affaires » posent de plus en plus de problèmes. Le tourisme devient alors une affaire de couple et les femmes, notamment les jeunes femmes, vont être les plus nombreuses à voyager à l'étranger.

113 Les études montrent comment la diminution du nombre des touristes masculins dans les destinations traditionnellement réputées pour le tourisme du sexe s'accompagne d'une croissance rapide d'immigrantes originaires de ces pays, tout d'abord de Corée du Sud et de Taiwan - où elles viennent avec des visas d'étudiants leur permettant de travailler légalement comme « hôtesses » quatre heures par jour - puis d'Asie du SudEst. Les flux migratoires laissent apparaître une nette sur-représentation des populations féminines de moins de trente ans ${ }^{24}$, phénomène appelé localement de «Japayuki-san » (pour qualifier l'émigration de ces femmes étrangères venues travailler comme prostituées) (Sasaki, 1991).

114 Ces migrations s'inscrivent dans le prolongement de pratiques touristiques, le transfert d'activités déjà exercées dans le lieu touristique est alors effectué dans une logique économique.

Le lieu touristique : une plate-forme pour de nouvelles logiques migratoires

Lieux de contacts et de passages privilégiés, dans un contexte de fermeture des frontières, les lieux touristiques constituent des plates-formes qui reçoivent des flux variés de touristes et de travailleurs à partir desquelles s'établissent de nouvelles trajectoires migratoires.

116 Ces lieux permettent également de souligner la difficulté de contrôler les mobilités des hommes (article de Bernard dans ce dossier). Des pouvoirs décrètent l'établissement de lieux dits «touristiques » à partir desquels ils pensent maîtriser les déplacements des hommes. Par la création de nouveaux emplois, des schémas de développement imaginent naturellement fixer une population localement; or le fonctionnement des lieux met en évidence des flux migratoires à l'échelle nationale ou régionale qui vont à l'encontre du projet initial. Il ne suffit pas de créer des emplois touristiques pour que la population résidente révèle une soudaine "vocation ». Créer des emplois ne suffit donc pas pour décider des trajectoires migratoires et professionnelles des individus. Les mesures incitatives favorisent le projet politique mais seules des mesures de contrainte pourraient répondre aux souhaits autoritaires. Or elles ne peuvent pas s'inscrire dans le cadre d'un marché touristique ouvert à tous ${ }^{25}$.

117 De la même manière, les bilans touristiques sont rarement à la hauteur des espoirs affichés. Là aussi, le processus est plus complexe, on ne décrète pas «un lieu touristique» sur la base d'une stratégie de mise en valeur des «ressources potentielles ». Ce sont les touristes qui élisent les lieux et en font des lieux touristiques ; des friches d'hôtels et de ports de plaisance dans les îles de la Mer de Chine et des mers du Sud sont là pour nous rappeler qu'il n'est pas facile de manipuler des masses de vacanciers. Ces populations ont leur propre logique, leur circulation dans l'espace progresse entre des lieux proches, plus familiers, et d'autres plus lointains. Ces itinéraires circulatoires individuels sont d'une grande complexité.

Conclusion 
118 Le tourisme développe de l'emploi localement mais il est illusoire de croire qu'il va favoriser un plus grand enracinement de la population locale. Le tourisme relève des mobilités par excellence, non seulement il résulte de l'arrivée de touristes mais en plus il crée un appel de main-d'œuvre et suscite de fortes migrations de travail et de nouvelles circulations professionnelles. Le lieu touristique est peuplé "d'étrangers " attirés par cette nouvelle économie qui introduit une rupture brutale avec les équilibres précaires mais sécurisants des activités traditionnelles: ces étrangers ont plusieurs visages, les uns appellent les autres. Un espace anciennement enclavé devient un lieu-carrefour dans les nouvelles logiques migratoires internationales.

Ces migrations s'opèrent dans le cadre d'un marché de travail spécifique, d'un transfert de capital avec des investissements relativement faciles. Dans un premier temps, deux phases migratoires successives peuvent être distinguées :

120 - un premier appel de main-d'œuvre caractérise la création du marché, comme cela s'est produit dans les régions les plus anciennement touristiques et continue de se produire dans les lieux touristiques émergents. Ces travailleurs se partagent entre des emplois relevant de groupes internationaux avec leurs lots de sous-traitants locaux et une multitude d'activités improvisées au gré des opportunités ;

121 - la seconde vague migratoire s'insère dans un marché déjà établi. Elle concerne le renouvellement de la main-d'œuvre. Les rotations sont importantes tant au niveau des emplois les plus qualifiés qu'à celui des tâches les plus simples. La sous-traitance, associée à la mobilité des travailleurs et à la flexibilité, permet de garder une activité compétitive et rentable malgré la saisonnalité et les aléas de ces marchés émergents.

122 Le schéma des mobilités se complexifie ensuite malgré les logiques initiales diamétralement opposés de ces flux qui convergent dans ces lieux, reflet de la mondialisation, avec ses inégalités et tous ses espoirs, qui deviennent des creusets où se dessine de nouvelles «sociétés mobiles » (Stock, 2001). La connaissance de ces lieux, leur relative familiarité, peut les placer au cœur de nouveaux projets de vie: des touristes s'y installent durablement ou y vivent en alternance avec d'autres lieux, le transfert d'activité professionnelle peut être complet ou partiel avec une nouvelle articulation sur plusieurs lieux, la retraite constitue souvent une période privilégiée pour redéployer son espace de vie.

Les interrelations entre tourisme et migrations doivent être replacées dans le contexte de la mondialisation contemporaine et des processus politiques avec les règles concernant la circulation des hommes et des capitaux. Les relations sont de plus en plus imbriquées et l'espace est en train d'être utilisé d'une façon nouvelle et plus imaginative pour réunir la production et la consommation comme le soulignent Williams et Hall: "news trends in tourism and migration similarly make it difficult to determine 'where is home, what is our identity and why are we here?» (Williams and Hall, 2000).

124 Les mobilités multiformes actuelles brouillent les typologies les plus classiques, alors que les logiques des migrants s'inscrivent davantage dans de "sociétés circulatoires" (Tarrius, 2000), "pour une part grandissante de notre société, la plus favorisée et la plus mobile, la figure du continuum entre le routinier et l'exceptionnel tend à s'imposer » (Knafou, 2000). Entre tourisme et migration: se dessine un continuum de mobilité personnelle ou les deux variables migration et tourisme se fondent l'un en l'autre. (Williams and Hall, 2000). 


\section{BIBLIOGRAPHIE}

AITKEN Carmen, HALL C. Michael (2000) Migrant and foreign skills and their relevance to the tourism industry, Tourism geographies, 2,1, 66-86.

AGARWAL Sheela, BALL Rick, SHAW Gareth, Williams Allan M. (2000) The geography of tourism production : uneven disciplinary development? Tourism Geographies, 2, 3, pp. 241-263.

BALL R M (1988) Seasonality : a problem for workers in the tourism labour market? Service industries journal 8(4), pp. 501-513.

BALL R M (1989) Aspects of tourism, seasonality and local labour markets, Area, 21(1), pp. 35-45.

BASSAND Michel et KAUFMANN Vincent (2000) Mobilité spatiale et processus de métropolisation : quelles interactions?, in Michel Bonnet et Dominique Desjeux eds. Les territoires de la mobilité, Paris, PUF, pp. 129-140.

BELL Martin, WARD Gary (2000) Comparing temporary mobility with permanent migration, Tourism Geographies, 2, 1, pp. 87-107.

BENNETT B.C. (1979) Migration and rural community viability in central Dalmatia (Croatia), Yugoslavia, Papers in Anthropology, 20(1), pp. 75-83.

BERRIANE Mohamed (1983) Tourisme et emploi : le cas d'Agadir, Revue de Géographie du Maroc, 7, pp. 21-33.

BERRIANE Mohamed (1991) Tourisme national et migrations de loisirs au Maroc, Rabat, Faculté des Lettres et des Sciences Humaines, Série « Thèses et Mémoires ", 500 p.

BOUBAKRI Hassen (1996) Les entrepreneurs migrants du sud-est tunisien, in J. Césari ed., Réseaux transnationaux entre l'Europe et le Maghreb, Rapport de recherche pour la Commission des Communautés Européennes, vol 1, pp. 17-79, vol 2, pp.3-58 et pp. 192-212.

BRAS Karin, DAHLES Heidi (1999) Massage, Miss ? Women Entrepreneurs and beach Tourism in Bali, in Heidi Dahles and Krain Bras eds., Tourism and Small Entrepreneurs. Development, National Policy, and Entrepreneurial Culture : Indonesian Cases, New York, Cognizant Communication Corporation, pp. 35-51.

BRAS Karin, DAHLES Heidi (1999) Pathfinder, Gigolo, and Friend : Diverging Entrepreneurial Stratégies of Tourist Guides on Two Indonesian Islands, in Heidi Dahles and Karin Bras eds., Tourism and Small Entrepreneurs. Development, National Policy, and Entrepreneurial Culture : Indonesian Cases, New York, Cognizant Communication Corporation, pp. 128-145.

BRITTON S. (1989) Tourism, dependency and developement. A mode of analysis, in T.V. Singh, H.L. Theuns and F.M. Go eds., Towards appropiate tourism, Frankfurt am Main : Peter Lang, pp. 93-116.

BRITTON S. (1993) Tourism, dependency and development. A mode of analysis, in D. Pearce and R.W. Butler eds., Tourism research. Critiques and challenges, Londres et New York, Routledge, pp. 155-172.

BRUNEAU Michel (1995) Espaces et territoires de diasporas, in Michel Bruneau ed. Diasporas, Montpellier, RECLUS, p. 6-23.

BULLER H., HOGGART K. (1994) Vers une campagne européenne : les Britanniques en France rurale, L'Espace Géographique, n³, pp. 263-273. 
CASTLES M, MILLER M J (1993) The Age of Migration, London : Macmillan.

CAVACO Carminda (1993) A place in the sun : return migration and rural change in Portugal, in Russell King ed. Mass Migrations in Europe : the Legacy and the Future, London, Belhaven, pp.

174-191.

CAZES Georges (1998) Les mobilités touristiques internationales, in Rémy Knafou ed. La Planète «nomade ». Les mobilités géographiques aujourd'hui, Belin, pp. 77-91.

CHARMES J, DABOUSSI R, LEBON A (1993) Population, employement and migration in the countries of the mediterranean basin, Geneva, International Laboure office, Mediterranean Information Exchange System on International migration and Employment, Paper 93/1E.

CLAVAL Paul (1995) The Impact of Tourism on the Restructuring of European Space, in Allan M. Williams and Armando Montanari eds., European Tourism Regions, Spaces and Restructuring. Restructuring in Europe, John Wiley and Sons Ltd, pp. 247-263.

CONNELL John (1987) Paradise Left? Pacific Island Voyagers in the Modern World, in James T. Fawcett and Benjamin V. Cariño eds. Pacific Bridges. The new immigration from Asia and the Pacific Islands, New York, Center for Migration Studies, pp. 375-404.

COURGEAU Daniel (1988) Méthodes de mesure de la mobilité spatiale, Paris, Ed de l'INED.

CRANG Ph.(1997) Performing the tourist product, in Ch. Rojek and J Urry eds., Touring cultures. Transformations of travel and theory, Londres, Routledge, pp. 137-154.

CRIBIER Françoise (1969) La grande migration d'été des citadins en France, CNRS, 403 p.

DABLES Heidi (1999a) Tourism and Small Entrepreneurs in Developing Countries : A Theorical Perspective in Heidi Dahles and Karin Bras eds., Tourism and Small Entrepreneurs. Development, National Policy, and Entrepreneurial Culture : Indonesian Cases, New York, Cognizant Communication Corporation, pp. 1-19.

DAHLES Heidi (1999b) Small Businesses in the Indonesian Tourism Industry : Entrepreneurship or Employement?, in Heidi Dahles and Karin Bras eds., Tourism and Small Entrepreneurs. Development, National Policy, and Entrepreneurial Culture : Indonesian Cases, New York, Cognizant Communication Corporation, pp. 20-34.

DESJEUX Dominique et BONNET Michel (2000) Les significations multiples de la mobilité, in Michel Bonnet et Dominique Desjeux eds. Les territoires de la mobilité, Paris, PUF, pp. 15-19.

DEWAILLY Jean-Michel et FLAMENT Emile (1993) Géographie du tourisme et des loisirs, Paris, SEDES, $287 \mathrm{p}$.

DOMENACH Hervé et PICOUET Michel (1987) Le caractère de réversabilité dans l'étude des migrations, Population, $\mathrm{n}^{\circ}$ 3, p. 469-484.

DUHAMEL Philippe (1997) Les résidents étrangers européens à Majorque (Baléares). Pour une analyse de la transformation des lieux touristiques, Thèse, Paris VII, $750 \mathrm{p}$.

DUMONT Gérard-François (1995) Les migrations internationales : les nouvelles logiques, Paris, SEDES, $223 \mathrm{p}$.

EATON M. (1995) British expatriate service provision in Spain's Costa del Sol, Services industries journal, 15, pp. 251-266.

GUENGANT Jean-Pierre (1996) Migrations internationales et développement : les nouveaux paradigmes, REMI, 12, 2, pp. 107-121. 
GUENGANT Jean-Pierre (1998) Migrations Internationales Sud-Nord et Développement du Sud, Hommes et Migrations, 1214, pp. 17-27.

GUILLON Michelle et SZTOKMAN Nicole (2000) Géographie mondiale de la population, Paris, Ellipses, $284 \mathrm{p}$.

HAMMAR T. (1997) International migration, immobility and development, New-York, Berg Oxford, 316 p.

HUETZ de LEMPS Christian (1989) Un exemple de macrocéphalie touristique : les îles Hawaii, in Îles et tourisme en milieu tropical et subtropical, Bordeaux, CEGET, n 10, pp. 195-222.

JORDAN F (1997) An occupational hazard ? Sex segregation in tourism employment, Tourism management 18(8), pp. 525-534.

KANG Sophie Kyung-Mi, PAGE Stephen J. (2000) Tourism, migration and emigration : travel patterns of Korean-New Zealanders in the 1990s, Tourism Geographies, 2,1, pp. 50-65.

KING Russell (1995) Tourism, labour and International Migration, in Allan M. Williams and Armando Montanari Eds., European Tourism Regions, Spaces and Restructuring. Restructuring in Europe, John Wiley and Sons Ltd, pp. 177-190.

KING Russell ( 1984) Population mobility : emigration, return migration and internal migration, in Allan M. Williams ed., Southern Europe transformed : political and economic change in Greece, Italy, Portugal and Spain, London, Harper and Row, pp. 145-178.

KIM K. (1994) The political economy of foreign investment in Hawai'i, in A. Ibrahim ed. Social Process in Hawai'i, Honolulu, University of Hawai'i, pp. 40-55.

KNAFOU Rémy et alii (1997) Une approche géographique du tourisme, L’Espace Géographique, n 3, pp. 193-204.

KNAFOU Rémy (2000) Les mobilités touristiques et de loisirs et le système global des mobilités, in Michel Bonnet et Dominique Desjeux eds. Les territoires de la mobilité, Paris, PUF, pp. 85-94.

KONTOGEOGOPOULOS N. (1998) Accomodation employment patterns and opportunities, Annals of trourism research 25, pp. 314-339.

KRITZ Mary M., LIM Lin Lean, ZLOTNIK Hania eds. (1992) International Migration System, Oxford, Clarendon Press, 354 p.

LEVY Jacques (1998) Nous habitons des lieux multiples, in Rémy Knafou ed. La Planète "nomade». Les mobilités géographiques aujourd'hui, Belin, pp. 193-197.

LEVY Jacques (2000) Les nouveaux espaces de la mobilité, in Michel Bonnet et Dominique Desjeux eds. Les territoires de la mobilité, Paris, PUF, pp. 155-170.

MADDEN L (1999) Making money in the sun : the development of British and Irish owned business in the Costa del Sol, Brighton, University of Sussex, Research papers in geography $36 \mathrm{p}$.

MA MUNG Emmanuel, DORAÏ Kamel, HILY Marie-Antoinette (1998) La circulation migratoire : bilan des travaux, Migrations-Etudes, $\mathrm{n}^{\circ} 84,12 \mathrm{p}$.

MENDONZA E. (1982) Benefits of migration as a personal strategy in Nazare, Portugal, International migration review, 16(3), pp. 635-645.

MINERBI Luciano (1994) Sustainability versus growth in hawai'i, in I. Aoude ed. Social Process in Hawai'I, Honolulu, University of Hawai'i, vol. 35, pp. 145-160. 
MINERBI Luciano (1996) Hawai'i, in C. Michael Hall and Stephen Page eds. Tourism in the Pacific. Issues and cases, Londres, International Thomson Business Press, pp. 190-204.

MONTANARI Armando (1995) The Mediterranean Region : Europe's Summer Leisure Space, in Allan M. Williams and Armando Montanari Eds., European Tourism Regions, Spaces and Restructuring. Restructuring in Europe, John Wiley and Sons Ltd, pp. 41-65.

MONTANARI Armando, CORTESE A. (1993) Third world immigrants in Italy, in Russell King ed., Mass migration in Europe : The legacy and the future, London : Belhaven press, pp. 275-292.

MÜLLER Dieter K. (1999) German Second Homes Owners in the Swedish Countryside: On the Internationalization of the Leisure Space, Dept. of Social and Economic geography, Umea, Sweden.

MUROI Hisae and SASAKI Naoko (1997) Tourism and prostitution in Japan, in M. Thea Sinclair ed. Gender, Work and Tourism, Londres, Routledge, pp.180-219.

NOIN Daniel (1995) Géographie de la population, Masson, 281 p.

O’BRIEN K. (1998) The European business travel market, Travel and Tourism Analyst, 4, pp. 37-54.

O.M.T. (2000) Faits Saillants 2000, Madrid, OMT, 28 p.

O'REILLY K (1995) A new trend in European migration: contemporary British migration to Fuengirola, Costa del Sol, Geographical viewpoint, 23, pp. 25-36.

PHILLIPS M. (1993) Rural gentrification and the processes of class colonization, Journal of Rural Studies, 9, 2, pp. 123-140.

RETAILLÉ Denis (1998) Concepts du nomadisme et nomadisation des concepts, in Rémy Knafou ed. La Planète « nomade ». Les mobilités géographiques aujourd'hui, Belin, p. 37-58.

SALVÀ TOMÀS Pere (1991) La population des îles Baléares pendant 40 ans de tourisme de masse (1950-1990), Méditerranée, 1, pp. 7-14.

SASAKI S. (1991) Aija kara Fuko Kaze (Wind from Asia), Tokyo, Asahi Shimbun.

SESSA A. (1983) Manuel of Tourism Economics, Rome, CATAL.

SINGARAVELOU (1989) Le rôle du tourisme dans l'économie mauricienne, in Îles et tourisme en milieux tropical et subtropical, Bordeaux, CEGET, n 10, pp. 265-278.

SIMON Gildas (1995) Géodynamique des migrations internationales, Paris, PUF, 429 p.

SIMON Gildas (1997) Mondialisation et brouillage des schémas migratoires, Migrations internationales, $\mathrm{n}^{\circ} 2$, pp. 9-14.

SIMON Gildas (1998) La planétarisation des migrations internationales, in Rémy Knafou ed. La Planète «nomade ». Les mobilités géographiques aujourd'hui, Belin, pp. 59-76.

SMITH Stephen (1997) Tourism in industrialized nations, in Salah Wahab and John J. Pigram Tourism, Development and Growth, Routledge, pp. 147-163.

STOCK Mathis (2001) Mobilités géographiques et pratiques des lieux. Etude théorico-empirique à travers deux lieux touristiques anciennement constitués : Brighton and Hove (Royaume-Uni) et GarsmischPartenkirchen (Allemagne), Thèse de géographie, Université de Paris 7, 663 p.

TARRIUS Alain (1989) Anthropologie du mouvement, Caen, Paradigme, $192 \mathrm{p}$.

TARRIUS Alain (1992) Les Fourmis d'Europe : migrants riches, migrants pauvres et nouvelles villes, Paris, l'Harmattan, $210 \mathrm{p}$. 
TARRIUS Alain (2001) Au delà des Etats-nations ; des sociétés de migrants, Revue Européenne des Migrations Internationales, vol 17, $\mathrm{n}^{\circ}$ 2, pp. 37-61.

TER STEEGE Karin, STAM Sandra and BRAS Karin (1999) Mountain Guides in Lombok : Pathfinders up Gunung Rinjani, in Heidi Dahles and Karin Bras eds., Tourism and Small Entrepreneurs. Development, National Policy, and Entrepreneurial Culture : Indonesian Cases, New York, Cognizant Communication Corporation, pp. 112-127.

THUMERELLE Pierre-Jean (1986) Peuples en mouvement. La mobilité spatiale des populations, Paris, SEDES, $325 \mathrm{p}$.

THUMERELLE Pierre-Jean (2001) Hommes et espace : indépendance et interdépendance, in Pierre-Jean Thumerelle ed., Explications en géographie. Démarches, stratégies et modèles, Paris, SEDES, pp. 113-126.

VAN GEMERT Hanneke, VAN GENUGTEN Esther, DAHLES Heidi (1999) Tukang Becak : the Pedicap Men of Yogyakarta, in Heidi Dahles and Karin Bras eds., Tourism and Small Entrepreneurs. Development, National Policy, and Entrepreneurial Culture : Indonesian Cases, New York, Cognizant Communication Corporation, pp. 97-111.

VANHOVE Norbert (1997) Mass tourism : benefits and costs, in Salah Wahab and John J. Pigram eds., Tourism, Development and Growth, Routledge, pp. 50-77.

VERQUIN Béatrice (1998) La mondialisation de l'économie et la mise en place d'un nouvel espace migratoire français, in Rémy Knafou ed. La Planète «nomade ». Les mobilités géographiques aujourd'hui, Belin, pp. 175-192.

VIARD Jean (1994) La Société d'archipel ou Les Territoires du village global, Les Editions de l'Aube, 124 p.

WARNES M.A. (1994) Permanent and seasonal international retirement migration : the prospects for Europe, Netherlands geographical Studies, 173, pp. 69-81.

WILLIAMS Allan M. (1995) Capital and transnationalisation of tourism , in Armando Montanari and Allan M. Williams eds., European tourism : regions, spaces and restructuring, Chichester, JohnWiley and Sons, pp. 163-176.

WILLIAMS Allan M. and MONTANARI Armando (1995) Tourism and Economic Restructutring in Europe, in Allan M. Williams and Armando Montanari eds., European Tourism Regions, Spaces and Restructuring. Restructuring in Europe, John Wiley and Sons Ltd, pp. 1-15.

WILLIAMS Allan M., HALL C. Micheal (2000) Tourism and migration : new relationships between production and consuption, Tourism Geographies, 2, 1, pp.5-27.

WILLIAMS Allan M., KING Russell, WARNES Anthony, PATTERSON Guy (2000) Tourism and international retirement migration : new forms of an old relationship in southern Europe, Tourism Geographies, 2,1, pp. 28-49.

YOUELL R. (1998) Tourism : an introduction. Harlow, Longman.

ZELINSKY W. (1995) The rowing palate : North America's ethnic resaturant cuisines, Geoforum, 16, 1 , pp. 51-72. 


\section{NOTES}

1. -En 1998, les recettes liées au tourisme, y compris celles réalisées par le transport international des passagers représentent, avec 532 milliards de dollars US, $8 \%$ des recettes mondiales d'exportation de biens et de services (OMT, 2001).

2. -Contrairement à l'approche proposée par Cazes (1998: 77).

3. -La Thaïlande offre désormais cette possibilité aux jeunes retraités japonais qui désirent s'installer sur ses côtes, de telles pratiques renvoient à la multi-résidence. 4. -Certaines limites d'ordre statistique doivent être soulignées : les définitions des organismes officiels sont trop générales. De nombreux autres déplacements sont additionnés aux déplacements touristiques. En fait, tout déplacement qui ne relève pas d'une migration avec un visa de travail est comptabilisé comme touristique (voyage d'affaires, déplacement pour raisons médicales ou religieuses, visites familiales). Selon les enquêtes de l'Organisation Mondiale du Tourisme, les visiteurs qui se déplacent strictement " pour le plaisir, pour se divertir ou pour leurs vacances ", donc les touristes au sens strict, ne représentent que $62 \%$ des personnes ainsi recensées. Les autres motifs de voyage se partagent entre affaires et motifs professionnels (18\%) et santé, religion, visites à des parents et amis (20\%). Cette confusion permet d'entretenir des résultats très flatteurs pour les pouvoirs jouant la carte du tourisme. Cependant ces limites n'enlèvent rien à l'ampleur croissante des pratiques touristiques internationales.

5. - Comme les attentats du 11 septembre 2001, à la fin de la saison.

6. -Entre 1993 et 2000, deux mille migrants clandestins ont péri aux frontières de l'Europe (source Le Monde, 23 juin 2000). Cf. le site d'United for Intercultural Action qui actualise la liste des disparus www.united.non-profit.nl)

7. -Cf. l'affaire Tampa pendant l'été 2001 qui rompt avec l'image de l'Australie accueillante des Jeux Olympiques 2000. Les boat-people qui transitent dans les eaux indonésiennes sont renvoyés par les autorités australiennes vers la Nouvelle-Zélande, puis la Papouasie-Nouvelle-Guinée, puis transportés sur les îles Cocos, puis sur l'île Nauru.

8. -Par lieux touristiques nous entendons des lieux marqués par une présence significative de touristes, les logiques du marché touristique dominent l'économie locale, parfois de façon exclusive.

9. -Les cinq principaux pays d'accueil du Nord (États-Unis, Espagne, France, Italie et Royaume-Uni) totalisent $41 \%$ des recettes, dont 17,6 \% pour les seuls États-Unis en 2000.

10. -En 1950, les quinze premiers pays touristiques polarisaient la quasi-totalité des flux internationaux contre seulement les deux tiers aujourd'hui. De nouvelles destinations s'imposent en Asie orientale (Chine, Thaïlande, Malaysia) en Europe centrale et orientale (Fédération de Russie : 18,5 millions 1999, Pologne : 18, République Tchèque : 16 , Hongrie $: 12,9)$ où les situations semblent moins propices. Les positions des destinations africaines sont plus modestes (4,9 millions en Tunisie, 3,8 au Maroc, presque 1 au Kenya et moins de 400000 au Sénégal).

11. -Soit 14,1 millions $\$$ US de recettes touristiques en 1999 pour la Chine continentale, 7,9 millions pour Hong Kong et 3 millions pour Macao.

12. $-7,5$ millions $\$$ US de recettes.

13. -La part des recettes touristique représente $40 \%$ du PNB aux Bahamas (pour 1,5 million de visiteurs), $50 \%$ à Sainte-Lucie (270 000visiteurs) $70 \%$ dans les Iles Vierges 
britanniques (300 000 visiteurs) et jusqu'à $85 \%$ aux Maldives (430 000 visiteurs) et $87 \%$ à Antigua (250 000 visiteurs).

14. -Comme l'illustre O'Brien (1998) à travers le cas des transports aériens avec l'Alliance globale de British Airways qui associe Qantas, Japan Airlines, Canadian Airlines, US Airlines, Polish Airlines et Finnair.

15. -Sur l'île Maurice, dès 1987, 6000 emplois directs et 16000 indirects sont recensés dans le tourisme.

16. -Dans les années quatre-vingts, Berriane (1983) note que dans la région d'Agadir moins de 20 \% des employés des hôtels de luxe et des villages de vacances sont originaires de la région.

17. -De tels parcours sont classiques en Asie de Sud-Est où l'employé débute dans des petites structures éparses sur les côtes thaïlandaises ou des îles du golfe du Siam pour se diriger vers les hôtels des resorts réputés (comme Nusa Dua à Bali), puis s'orienter ensuite vers les hôtels des capitales de la région et principalement vers Singapour. La sortie peut se traduire par la création d'une petite structure sur fonds propres à Bali par exemple.

18. -Lors de la crise asiatique (1997), le marché touristique régional a immédiatement été touché, de la même manière il s'est rétracté momentanément pendant l'automne 2001. La croissance reprend rapidement son rythme. Ces crises ponctuelles servent souvent d'alibi pour annoncer des difficultés (souvent anciennes), licencier et introduire plus de flexibilité.

19. -La colonisation économique japonaise commença au début des années soixante-dix (dès que le gouvernement japonais autorisa ce type d'investissement), elle s'est accentuée dans les années quatre-vingts, soutenue par la solidité du yen sur les marché internationaux (les investissement étaient de 1,5 milliards de dollars US en 1984 et de 9,3 en 1990).

20. -Pendant les années quatre-vingts, le coût de la vie a augmenté six fois plus vite que les revenus moyens, la part des populations vivant sous le seuil de pauvreté est de $11 \%$ en 1990 contre $8 \%$ en 1980.

21. -Cité par G. Cazes (1998), il s'agit d'une conférence intitulée « Tourism in Portugal : changing forms of internationalisation », présentée dans le cadre de Conference on Geography of leisure and Tourism, mai 1996.

22. -Cette réalité ne doit pas occulter l'existence de véritables touristes provenant de ces pays (appartenant aux élites, aux classes supérieures et parfois aux nouvelles classes moyennes). Un premier séjour à des fins touristiques peut aussi être l'occasion de construire un projet migratoire.

23. -C'est à cette époque que le gouvernement sud-coréen entreprit d'importants investissements touristiques sur l'île de Cheju, au sud du Japon. Cette île qui devait devenir la vitrine du pays connut ses premiers succès auprès de ces clientèles japonaises en voyage d'affaire.

24. -Par exemple, les jeunes femmes philippines (entre 20 et 29 ans) représentaient 76,9 du total des immigrants philippins en 1982, 86,6 \% en 1987, puis 88,9\% en 1992 (source : ministère de la Justice du Japon).

25. -Les forces birmanes ont actuellement plus d'aisance à développer de lourds travaux avec le recours au travail forcé, dans le cadre d'investissements relevant de partenariat avec des multinationales, qu'à développer un secteur touristique acceptable aux yeux des visiteurs critiques. 


\section{RÉSUMÉS}

Réalité économique établie, le marché touristique est au cœur de trajectoires migratoires variées et renouvelées dans un contexte international de plus en plus contraignant. Les lieux touristiques, anciens et émergents, constituent d'intéressants laboratoires pour analyser les logiques des mobilités contemporaines et leurs recompositions multiformes. Ils constituent des plates-formes qui reçoivent des flux de touristes et de travailleurs à partir desquelles s'établissent de nouvelles trajectoires migratoires. De ces points de rencontre entre des flux variés où se dessinent des interrelations, des chevauchements, des filiations.

Tourism, Labour, Migration: Relationships and Human Mobility.-- A significant economic reality, the tourism market is at the origin of new and varied migratory paths in an increasingly restrictive international context. Established and emerging tourist destinations provide interesting laboratories in which to analyse the logic of the multiple forms of contemporary mobility. They constitute platforms that receive flows of tourists and workers from which new migratory paths are forged. At the meeting points between various flows, interrelationships, overlappings, and new filiations take shape.

Turismo, trabajo, migración : interrelaciones y lógicas de movilidad. -- Como realidad económica consolidada, el mercado turístico se encuentra en el centro de trayectorias migratorias diversas y renovadas en un contexto internacional cada vez más complejo. Las zonas turísticas -las clásicas y las emergentes- constituyen interesantes laboratorios a la hora de analizar la lógica de las movilidades contemporáneas y sus múltiples recomposiciones. Estos lugares, que reciben flujos de turistas y trabajadores, son una especie de plataformas que generan nuevas corrientes migratorias. En estos puntos de encuentro entre flujos diversos surgen interrelaciones, imbricaciones, filiaciones.

\section{INDEX}

Mots-clés : flux, travail, tourisme, mobilité, trajectoire migratoire

\section{AUTEUR \\ OLIVIER DEHOORNE}

Maître de conférences, Migrinter (Université de Poitiers-CNRS), 99 avenue du Recteur Pineau, 86000 Poitiers, France, et MIT, Université de Paris VII 